\title{
Consistency in NLO analyses of inclusive and semi-inclusive polarized DIS data
}

\author{
G.A. Navarro, R. Sassot ${ }^{a, *}$ \\ Departamento de Física, Facultad de Ciencias Exactas y Naturales, Universidad de Buenos Aires, Pabellón I, \\ Ciudad Universitaria, (1428) Buenos Aires, Argentina
}

Received: 2 December 2002 / Revised version: 6 February 2003 /

Published online: 31 March 2003 - (c) Springer-Verlag / Società Italiana di Fisica 2003

\begin{abstract}
We perform a detailed study of the consistency between different sets of polarized deep inelastic scattering data and theory, from the standpoint of a next to leading order QCD global analysis, and following the criteria proposed by Collins and Pumplin. In face of recent suggestions that challenge the usual assumption about parent parton spin independence of unpolarized fragmentation functions, we specially focus on polarized semi-inclusive data.
\end{abstract}

\section{Introduction}

Ever since the renewed burst of interest in the spin structure of the nucleon, triggered fifteen years ago by the measurement of the proton spin dependent deep inelastic scattering structure function $g_{1}^{p}$ by the EMC experiment [1], polarized deep inelastic scattering has evolved into a very prolific field for combined theoretical and experimental efforts [2]. As a result of this activity, an increasingly precise QCD improved partonic description of polarized nucleons has emerged, phrased in terms of more refined and exhaustive extractions of polarized parton distributions.

Indeed, the number of QCD analyses of polarized inclusive deep inelastic scattering (DIS) data at next to leading order (NLO) accuracy has rapidly grown in the last few years [3]. In some cases these studies also take into account polarized semi-inclusive deep inelastic scattering (SIDIS) data [4-6]. The usual outcome from these analyses are different sets of parton distributions which reproduce fairly well most data sets within the quoted errors, with overall $\chi^{2}$ values pretty close to the number $N$ of degrees of freedom (d.o.f.) as required by the familiar "hypothesis testing criterion".

Although they are the apparent fulfillment of this requirement, the resulting parton distributions not only suffer from large uncertainties, but in some cases small subsets of inclusive data are badly fitted. These facts point to the need of a more stringent criterion for assessing the goodness of a particular fit, and also the compatibility between the data sets.

In the case of polarized semi-inclusive data, the motivation for a more careful analysis is twofold. On the one side, inclusive data do not provide enough information for

\footnotetext{
a e-mail: sassot@df.uba.ar

* Partially supported by Conicet and Fundación Antorchas
}

a complete separation of the quark and antiquark distributions of different flavors, so the analyses must rely either on SIDIS data or on some external assumption.

On the other side, the standard procedure used to analyze semi-inclusive data, in terms of polarized parton densities and unpolarized fragmentation functions, has been criticized, questioning, in the first place, the accuracy of the present generation of fragmentation functions [7], suggesting that the usual assumptions about the spin independence of a fragmentation process may not hold [8], and also addressing the issue of target fragmentation contributions [9].

Regarding the first issue, recently there has been an increasing amount of activity around the issue of the polarization of sea quarks in the proton [10]. This interest has been driven in part by the confirmation of the isospin symmetry breaking at sea quark level in unpolarized DIS [11], by the level of refinement attained in a QCD global analysis of the spin dependent data [3], and by the expected precision of forthcoming polarized semi-inclusive measurements, polarized Drell-Yan dilepton production, and prompt photon production [12].

Moreover, in spite of the encouraging achievements in polarized semi-inclusive measurements in the few last years $[13,14]$, which have shown to be in good agreement with the inclusive data [4], the available data fail to constrain unambiguously the polarization of sea quarks in the proton when included in global QCD fits performed following just the hypothesis testing criterion [5].

In face of this situation, it is worthwhile assessing the goodness of global fits to the polarized data, involving both inclusive and semi-inclusive measurements, with more stringent criteria, in line of what has been proposed in [15], and applied to LO unpolarized sets of data in [16]. The main point of this approach is to apply the parame- 
ter fitting criterion to subsets of data in the global fit. In this way we assess features as the degree of compatibility between subsets of data, the impact of specific data in certain parameters of the fit and the overall consistency of the global fit. The occurrence of such an inconsistency may hint at the non-validity of assumptions or other inputs of the theoretical calculation, besides an unexpected error source in the experiment.

In the following, after defining our conventions and sketching the computation of the relevant observables, we present a global fit of polarized inclusive and semiinclusive data exploring the dependence of the overall $\chi_{\text {tot }}^{2}$ of the fit on the respective $\chi_{i}^{2}$ for each particular experiment by means of the Lagrange multiplier method [19]. The analysis is performed in two stages. In the first one we restrict the analysis to inclusive data, and to those parton densities which can be extracted there. In the second stage we add semi-inclusive observables and discriminate between valence and sea quark densities of different flavors, but at variance with [5], where semi-inclusive data were only allowed to fix the sea quark polarization, here we leave all the distributions free.

In order to circumvent the extremely time-consuming convolutions integrals characteristic of the semi-inclusive observables at NLO, we apply the double Mellin transform approach, developed recently in [6]. This new approach has produced results in complete agreement with those obtained with the convolution method, but obtained considerably faster.

As a result of our analysis, we find that the parameter fitting criterion shows a reasonably good level of internal consistency between the inclusive measurements. The addition of SIDIS data sets leads to global fits with $\chi^{2} /$ d.o.f. values fairly close to unity; however the increase of the statistical weight of these data in the fits show rather different consequences when either positively $\left(\sigma^{h+}\right)$ or negatively $\left(\sigma^{h-}\right)$ charged final state hadron data are considered. For $\sigma^{h-}$ data, the increase in weight does not significantly modify those parton distributions coming from inclusive sets, suggesting consistency between the different data sets and also between the data and the theoretical framework. However, the analysis of the $\sigma^{h+}$ data shows a sizable degree of conflict with the rest of the fit.

\section{Framework}

Throughout the present analysis, we follow the same conventions and definitions for the polarized inclusive asymmetries and parton densities as in [5]. In the totally inclusive case, the spin dependent asymmetries are given by [2]

$$
\begin{aligned}
A_{1}^{N}\left(x, Q^{2}\right) & =\frac{g_{1}^{N}\left(x, Q^{2}\right)}{F_{1}^{N}\left(x, Q^{2}\right)} \\
& =\frac{g_{1}^{N}\left(x, Q^{2}\right)}{F_{2}^{N}\left(x, Q^{2}\right) / 2 x\left[1+R^{N}\left(x, Q^{2}\right)\right]},
\end{aligned}
$$

where the inclusive spin dependent nucleon structure function $g_{1}^{N}\left(x, Q^{2}\right)$ can be written at NLO as a convolution between polarized parton densities for quarks and gluons, $\Delta q_{i}\left(x, Q^{2}\right)$ and $\Delta g\left(x, Q^{2}\right)$, respectively, and coefficient functions $\Delta C_{i}(x)[17]$

$$
\begin{aligned}
g_{1}^{N}\left(x, Q^{2}\right)= & \frac{1}{2} \sum_{q, \bar{q}} e_{q}^{2}\left[\Delta q\left(x, Q^{2}\right)\right. \\
& +\frac{\alpha_{\mathrm{s}}\left(Q^{2}\right)}{2 \pi} \int_{x}^{1} \frac{\mathrm{d} z}{z}\left\{\Delta C_{q}(z) \Delta q\left(\frac{x}{z}, Q^{2}\right)\right. \\
& \left.\left.+\Delta C_{g}(z) \Delta g\left(\frac{x}{z}, Q^{2}\right)\right\}\right] .
\end{aligned}
$$

A more detailed discussion of these coefficient functions and their factorization scheme dependence can be found in [18]. $F_{1}^{N}\left(x, Q^{2}\right)$ is the unpolarized nucleon structure function that can be written in terms of $F_{2}^{N}\left(x, Q^{2}\right)$ and $R$, the ratio of the longitudinal to transverse cross section [2].

Analogously, for the semi-inclusive asymmetries we have

$$
\left.A_{1}^{N h}\left(x, Q^{2}\right)\right|_{Z} \simeq \frac{\int_{Z} \mathrm{~d} z g_{1}^{N h}\left(x, z, Q^{2}\right)}{\int_{Z} \mathrm{~d} z F_{1}^{N h}\left(x, z, Q^{2}\right)},
$$

where the superscript $h$ denotes the hadron detected in the final state, and the variable $z$ is given by the ratio between the hadron energy and that of the spectators in the target. The region $Z$, over which $z$ is integrated, is determined by kinematical cuts applied when measuring the asymmetries.

For the spin dependent structure function $g_{1}^{N}\left(x, Q^{2}\right)$ we use the NLO expression [20]

$$
\begin{aligned}
& g_{1}^{N h}(x, z, Q)=\frac{1}{2} \sum_{q, \bar{q}}^{2}\left[\Delta q\left(x, Q^{2}\right) D_{q}^{H}\left(z, Q^{2}\right)\right. \\
& +\frac{\alpha_{\mathrm{s}}\left(Q^{2}\right)}{2 \pi} \int_{x}^{1} \frac{\mathrm{d} \hat{x}}{\hat{x}} \int_{z}^{1} \frac{\mathrm{d} \hat{z}}{\hat{z}} \\
& \times\left\{\Delta q\left(\frac{x}{\hat{x}}, Q^{2}\right) \Delta C_{q q}^{(1)}\left(\hat{x}, \hat{z}, Q^{2}\right) D_{q}^{H}\left(\frac{z}{\hat{z}}, Q^{2}\right)\right. \\
& \quad+\Delta q\left(\frac{x}{\hat{x}}, Q^{2}\right) \Delta C_{g q}^{(1)}\left(\hat{x}, \hat{z}, Q^{2}\right) D_{\mathrm{g}}^{H}\left(\frac{z}{\hat{z}}, Q^{2}\right) \\
& \left.\left.\quad+\Delta g\left(\frac{x}{\hat{x}}, Q^{2}\right) \Delta C_{q g}^{(1)}\left(\hat{x}, \hat{z}, Q^{2}\right) D_{q}^{H}\left(\frac{z}{\hat{z}}, Q^{2}\right)\right\}\right],
\end{aligned}
$$

and in order to avoid the convolution integrals in $\hat{x}$ and $\hat{z}$ we switch to moment space in both variables as suggested in [6]. In moment space, the convolution integrals reduce products of the Mellin moments of the parton densities

$$
\Delta f_{i}^{n}\left(Q^{2}\right) \equiv \int_{0}^{1} \mathrm{~d} x x^{n-1} \Delta f_{i}\left(x, Q^{2}\right)
$$

the fragmentation functions

$$
\Delta D_{i}^{h m}\left(Q^{2}\right) \equiv \int_{0}^{1} \mathrm{~d} x x^{m-1} \Delta D_{i}^{h}\left(z, Q^{2}\right),
$$

and the double Mellin transform of the coefficient functions $\Delta C_{i j}^{(1)}\left(x, z, Q^{2}\right)$, defined by 


$$
\begin{aligned}
\Delta C_{i j}^{(1), n m}\left(Q^{2}\right) \equiv & \int_{0}^{1} \mathrm{~d} x x^{n-1} \\
& \times \int_{0}^{1} \mathrm{~d} z z^{m-1} \Delta C_{i j}^{(1)}\left(x, z, Q^{2}\right) .
\end{aligned}
$$

These coefficients can be written as [21]

$$
\begin{aligned}
& \Delta C_{q q}^{(1), n m}\left(Q^{2}\right)=C_{F}\left[-8-\frac{1}{m^{2}}+\frac{2}{(m+1)^{2}}+\frac{1}{n^{2}}\right. \\
& +\frac{(1+m+n)^{2}-1}{m(m+1) n(n+1)}+\left[S_{1}(m)+S_{1}(n)\right] \\
& \times\left\{S_{1}(m)+S_{1}(n)-\frac{1}{m(m+1)}-\frac{1}{n(n+1)}\right\} \\
& \left.+3 S_{2}(m)-S_{2}(n)\right] \text {, } \\
& \Delta C_{g q}^{(1), n m}\left(Q^{2}\right)=C_{F}\left[\frac{2-2 m-9 m^{2}+m^{3}-m^{4}+m^{5}}{m^{2}(m-1)^{2}(m+1)^{2}}\right. \\
& +\frac{2 m}{n(m+1)(m-1)}-\frac{2-m+m^{2}}{m(m+1)(m-1)(n+1)} \\
& -\frac{2+m+m^{2}}{m(m+1)(m-1)}\left[S_{1}(m)+S_{1}(n)\right] \\
& \left.-\frac{2}{(m+1) n(n+1)}\right] \\
& \Delta C_{q g}^{(1), n m}\left(Q^{2}\right)=T_{R} \frac{n-1}{n(n+1)} \\
& \times\left[\frac{1}{m-1}-\frac{1}{m}+\frac{1}{n}-S_{1}(m)-S_{1}(n)\right],
\end{aligned}
$$

where we have set the factorization and renormalization scales to $Q^{2}$. As usual, $C_{F}=4 / 3, T_{R}=1 / 2$, and

$$
S_{i}(n) \equiv \sum_{j=1}^{n} \frac{1}{j^{i}}
$$

For $u$ and $d$ quark plus antiquark densities at the initial scale $Q_{0}^{2}=0.5 \mathrm{GeV}^{2}$ we propose

$$
\begin{aligned}
& x(\Delta q+\Delta \bar{q}) \\
& \quad=N_{q} \frac{x^{\alpha_{q}}(1-x)^{\beta_{q}}\left(1+\gamma_{q} x^{\delta_{q}}\right)}{B\left(\alpha_{q}+1, \beta_{q}+1\right)+\gamma_{q} B\left(\alpha_{q}+\delta_{q}+1, \beta_{q}+1\right)},
\end{aligned}
$$

where $B(\alpha, \beta)$ is the standard beta function, while for strange quarks plus antiquarks we use

$$
x(\Delta s+\Delta \bar{s})=2 N_{s} \frac{x^{\alpha_{\mathrm{s}}}(1-x)^{\beta_{s}}}{B\left(\alpha_{\mathrm{s}}+1, \beta_{s}+1\right)},
$$

with a similar parametric form for gluons

$$
x \Delta g=N_{\mathrm{g}} \frac{x^{\alpha_{g}}(1-x)^{\beta_{g}}}{B\left(\alpha_{g}+1, \beta_{g}+1\right)} .
$$

The first moments of the quark densities $\delta q\left(N_{q}\right)$ are often related to the hyperon beta decay constants $F$ and $D$ through the $S U(3)$ symmetry relations

$$
\begin{gathered}
\delta u+\delta \bar{u}-\delta d-\delta \bar{d} \equiv N_{u}-N_{d} \\
=F+D=1.2573 \\
\delta u+\delta \bar{u}+\delta d+\delta \bar{d}-2(\delta s+\delta \bar{s}) \equiv N_{u}+N_{d}-4 N_{s} \\
=3 F-D=0.579 .
\end{gathered}
$$

Under such an assumption, the previous equations would strongly constrain the normalization of the quark densities. However, as we are not interested in forcing flavor symmetry, we leave aside that strong assumption and relax the symmetry relations introducing the two parameters $\epsilon_{\mathrm{Bj}}$ and $\epsilon_{S U(3)}$ respectively. These parameters account quantitatively for eventual departures from flavor symmetry considerations, including also some uncertainties on the low- $x$ behavior, and higher order corrections,

$$
\begin{gathered}
N_{u}-N_{d}=(F+D)\left(1+\epsilon_{\mathrm{Bj}}\right), \\
N_{u}+N_{d}-4 N_{s}=(3 F-D)\left(1+\epsilon_{S U(3)}\right),
\end{gathered}
$$

and we take these as a measure of the degree of fulfilment of the Bjorken sum rule [22] and the $S U(3)$ symmetry.

Equations (16) and (17) allow one to write the normalization of the three quark flavors in terms of $N_{s}, \epsilon_{\mathrm{Bj}}$, and $\epsilon_{S U(3)}$. Notice that no constraints have been imposed on the breaking parameters since we expect them to be fixed by data. The remaining parameters are constrained in such a way that positivity with respect to GRV98 parton distributions is fulfilled. These last parameterizations are used in order to compute the denominators of (1) and (3). This is particularly relevant at large $x$, and since no polarized data are available in that kinematical region, we directly fix the parameters $\beta_{u}=3.2, \beta_{d}=4.05$ and $\beta_{g}=6$ for the NLO sets in agreement with GRV98. Consistently with the choice for the unpolarized parton distributions, we use the values of $\Lambda_{\mathrm{QCD}}$ given in [23] to compute $\alpha_{\mathrm{s}}$ at NLO.

As antiquark densities we take

$$
x \Delta \bar{q}=N_{\bar{q}} \frac{x^{\alpha_{\bar{q}}}(1-x)^{\beta_{\bar{q}}}}{B\left(\alpha_{\bar{q}}+1, \beta_{\bar{q}}+1\right)},
$$

for the $\bar{u}$ and $\bar{d}$ quarks, and we assume $\bar{s}=s$ since the possibility of discrimination in the $s$ sector is beyond the precision of the data (as in the unpolarized case).

Fragmentation functions are taken from [24] and we also use the flavor separation criterion proposed there, which have shown to be in agreement with the most recent analysis [25].

The data sets analyzed include only the points with $Q^{2}>1 \mathrm{GeV}^{2}$ listed in Table 1, and totaling 137, 118, and 34 points, from proton, deuteron, and helium targets respectively, in the inclusive stage, plus 42, 24, and 18, from proton, deuteron, and helium targets respectively, in the second stage.

Regarding the fitting procedure, this is done minimizing the function $f\left(\lambda_{1}, \lambda_{2}, \ldots, \lambda_{n}\right)$ defined by

$$
f\left(\lambda_{1}, \lambda_{2}, \ldots, \lambda_{n}\right)=\sum_{i} \lambda_{i} \chi_{i}^{2},
$$


Table 1. Inclusive and semi-inclusive data used in the fit

\begin{tabular}{ccccc}
\hline Collaboration & Target & Final state & \# points & Refs. \\
\hline EMC & proton & inclusive & 10 & {$[1]$} \\
SMC & proton, deuteron & inclusive & 12,12 & {$[27]$} \\
E-143 & proton, deuteron & inclusive & 82,82 & {$[28]$} \\
E-155 & proton, deuteron & inclusive & 24,24 & {$[29]$} \\
Hermes & proton, helium & inclusive & 9,9 & {$[14]$} \\
E-142 & helium & inclusive & 8 & {$[30]$} \\
E-154 & helium & inclusive & 17 & {$[28]$} \\
\hline SMC & proton, deuteron & $h^{+}, h^{-}$ & 24,24 & {$[13]$} \\
Hermes & proton, helium & $h^{+}, h^{-}$ & 18,18 & {$[14]$} \\
\hline
\end{tabular}

where the sum runs over the data sets or experiments $i$ included in the fit. The parameters $\lambda_{i}$ are the Lagrange multipliers defined for each data set and which can be varied in order to produce different fits where the relative weight of a given set of data has been modified. $\chi_{i}^{2}$ is the contribution to the overall $\chi^{2}$ coming from the data set $i$.

As is well known, there are various alternatives for calculating these last contributions [32]. The simplest one, and the one most commonly used in fits to polarized data, adds the reported statistical and systematic errors in quadrature. This ignores the correlations between data points from the same measurements, but in many cases the full correlation matrices are not available.

As inclusive and semi-inclusive data are strongly correlated, and the correlation matrices are available, we have taken into account them, analyzing only the inclusive data for SMC and Hermes corresponding to "averaged" bins, for which the correlation matrices are defined. In the semiinclusive case we only consider in the fit the most precise data concerning the production of charged \pm hadrons (without identifying pions, kaons, or other particles individually).

Regarding the normalization uncertainties, in [5] it was found that the Hermes data were systematically below the best global fits, but allowing for a floating normalization factor to it in order to account for the relative normalization uncertainties, the $\chi^{2}$ values were considerably improved. In the following we allow this factor to be fixed by the global fit, finding the best fits for a $12 \%$ correction.

\section{Inclusive data}

In this section we present results from the first stage of our analysis, which only deals with inclusive data. It is customary in NLO fits to inclusive data to present several sets of parton distributions with different alternatives for the features that are poorly constrained by the data, such as the gluon or strange sea quark polarization. As we are mainly concerned in assessing the goodness of the fit between theory and data and the degree of internal consistency of the data, rather than covering the different scenarios for parton densities, we just explore the more
Table 2. $\chi_{i}^{2}(\lambda)$ and $\chi_{\mathrm{Inc}}^{2}(\lambda)$ for different data sets

\begin{tabular}{ccccccc}
\hline & \multicolumn{2}{c}{$\lambda=1$} & \multicolumn{2}{c}{$\lambda=20$} & \multicolumn{2}{c}{$\lambda=100$} \\
& $\chi_{i}^{2}$ & $\chi_{\text {Inc }}^{2}$ & $\chi_{i}^{2}$ & $\chi_{\text {Inc }}^{2}$ & $\chi_{i}^{2}$ & $\chi_{\text {Inc }}^{2}$ \\
\hline EMC-p & 4.49 & & 4.17 & 227.85 & 3.46 & 262.71 \\
SMC-p & 3.84 & & 3.32 & 225.21 & 3.20 & 226.71 \\
SMC-d & 14.44 & 12.81 & 245.92 & 12.10 & 264.77 \\
E-143-p & 60.70 & 60.22 & 224.83 & 60.21 & 224.95 \\
E-143-d & 83.38 & & 80.84 & 235.32 & 80.17 & 248.08 \\
E-142-n & 4.68 & 224.59 & 2.40 & 233.52 & 1.51 & 251.98 \\
E-155-p & 17.15 & & 16.24 & 227.03 & 16.23 & 233.55 \\
E-155-d & 17.10 & & 16.89 & 225.65 & 14.98 & 277.42 \\
E-154-n & 6.91 & & 4.19 & 229.16 & 3.97 & 232.02 \\
Hermes-p & 5.15 & & 4.30 & 225.60 & 2.83 & 234.42 \\
Hermes-He & 6.76 & & 6.39 & 228.31 & 5.67 & 248.09 \\
\hline
\end{tabular}

favored scenario of [5], which was labeled as "set $i$ " and has moderate gluon polarization.

In Fig. 1 we show the outcome of different global fits to all the available inclusive data. The plot gives the variation of the $\chi_{i}^{2}$ value of each experiment against the total $\chi^{2}$ value of the fit $\left(\chi_{\text {Inc }}^{2}\right)$. The first point to the left of each curve $\left(\lambda_{i}=1\right)$ corresponds to standard fits where no extra weight was given to any of the data sets. The parameters for this fit are presented in Table A1 in the appendix. Along the curves, the subsequent points come from fits where, following the Lagrange multiplier method explained in the previous section, increasing values of $\lambda_{i}$ have been given to a specific set of data, while keeping the other parameters $\lambda_{j}$ equal to 1 .

The normal expectation in a good fit to data sets that individually only determine a small fraction of the parameters is a monotonic decrease of a few units in the $\chi_{i}^{2}$ of the subset which has been subject to the increase in weight while $\chi_{\text {Inc }}^{2}$ varies in the range $N \pm \sqrt{2 N}$. One would also expect $\chi_{i}^{2}$ to approach a saturation point within an increase of $\chi_{\text {Inc }}^{2}$ smaller than $\sqrt{2 N}$.

As can be seen in Fig. 1, in all the cases, the curves show the features expected for consistent subsets of data, each one able to fix a limited number of parameters. The $\chi_{i}^{2}(\lambda)$ values of each experiment $i$ are shown in Table 2 .

Notice that the initial fall in the $\chi_{i}^{2}(\lambda)$ values with a very mild variation in $\chi_{\text {Inc }}^{2}$ illustrates the situation where a subset of the data would be able to effectively fix some parameters in the fit, in general agreement with the remaining data, if its statistical significance were increased. This is clearly the situation of the neutron and deuteron target data.

The standard $\lambda_{i}=1$ fit (solid line) can be seen in Fig. 2 together with the Hermes-p driven fit for $\lambda_{\text {Hermes }}=100$ (dashes) and that for E155 with $\lambda_{\mathrm{E} 155}=100$ (dots) for comparison. As can be noticed, the changes in the asymmetries due to the extra weight in these subsets of data are almost negligible, as can be expected from the moderate variations they produce in $\chi_{\text {Inc }}^{2}(\lambda)$. For these reasons 

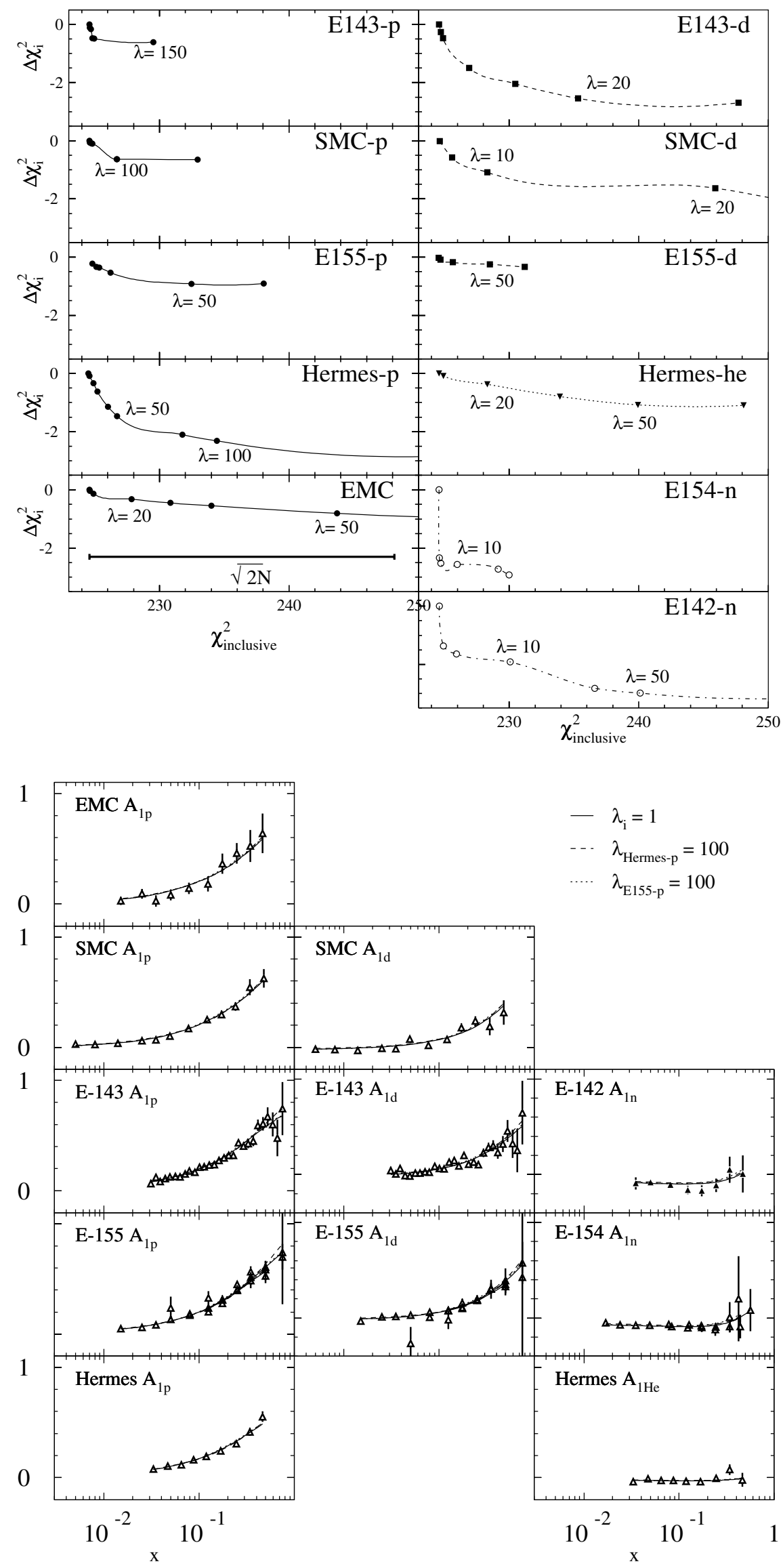

Fig. 1. $\chi_{i}^{2}(\lambda)$ against $\chi_{\text {Inc }}^{2}(\lambda)$ for all inclusive data sets
Fig. 2. Fit to inclusive data $(\lambda=1)$ together with the Hermes-p and E155-p driven fits with $\lambda=50$ 


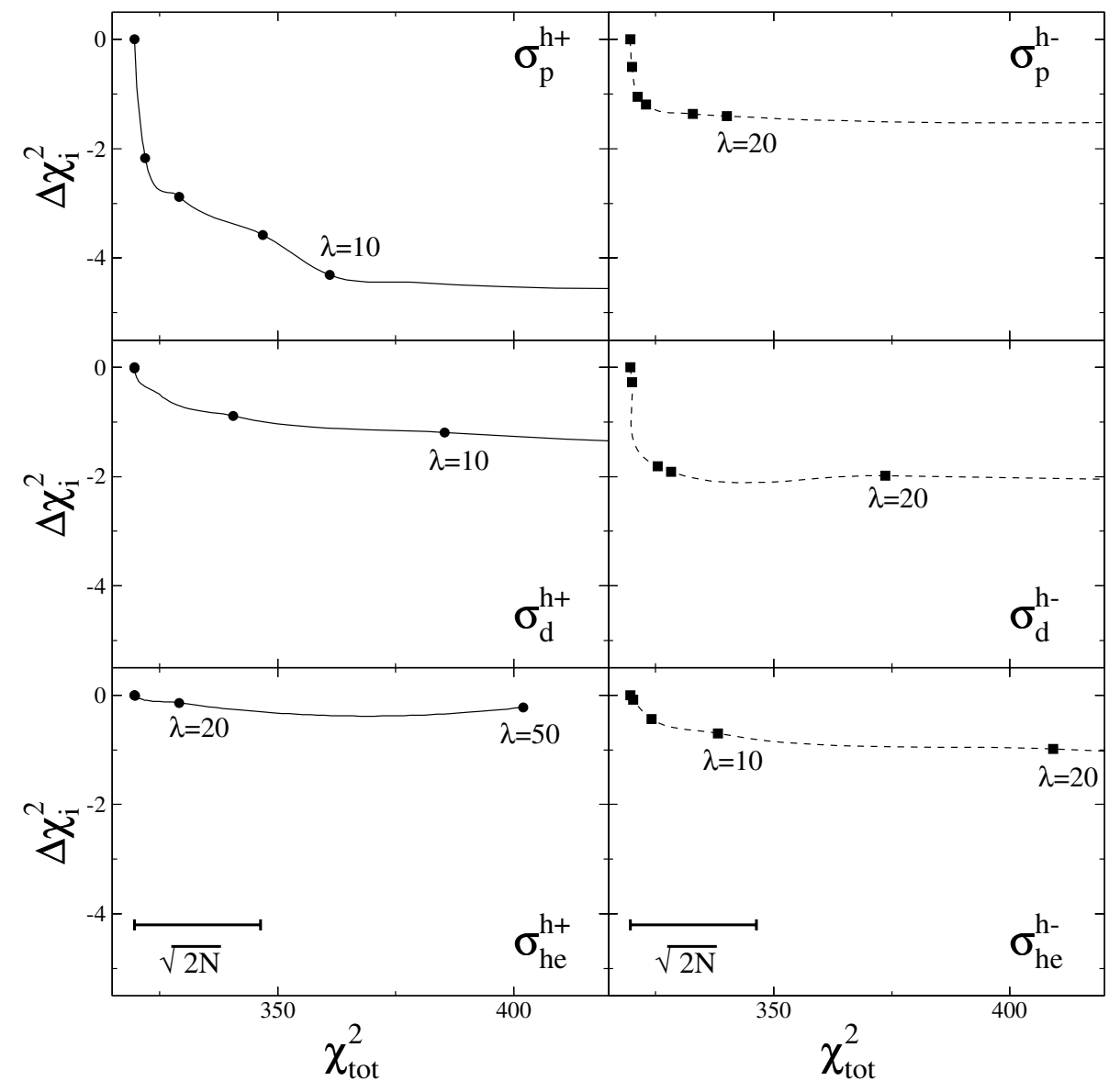

Fig. 3. $\chi_{i}^{2}(\lambda)$ against $\chi_{\text {tot }}^{2}(\lambda)$ for semiinclusive data sets we conclude that inclusive data are internally consistent and in excellent agreement with theory.

\section{Semi-inclusive data}

In this section we focus on the consequences of including SIDIS data in the global fits. These data allow one in principle to discriminate between light sea quark flavors, so the corresponding parton distributions are now parameterized and fitted. As we have anticipated, the inclusion of SIDIS data leads to acceptable fits according to the hypothesis testing criterion [5]; however the small statistical impact of SIDIS data relative to DIS data hinders definite conclusions on sea quark distributions.

A closer examination allowing for example increased weights in the different SIDIS data subsets, as we did for inclusive sets, reveals some interesting features, as shown in Fig. 3. Again, we have applied a $12 \%$ normalization factor to the Hermes data, and in order to simplify the analysis, we consider the SMC and Hermes proton target data together. Identical results are obtained if the data are discriminated for each experiment.

Although for most SIDIS data sets, $\chi_{i}^{2}(\lambda)$ reach their respective saturation values within a $\sqrt{2 N}$ shift in $\chi_{\text {tot }}^{2}$ as required for overall consistency, the data coming from positively charged hadroproduction on proton targets $\left(\sigma_{p}^{h+}\right)$ seem to lay in the borderline, with a fall of several units

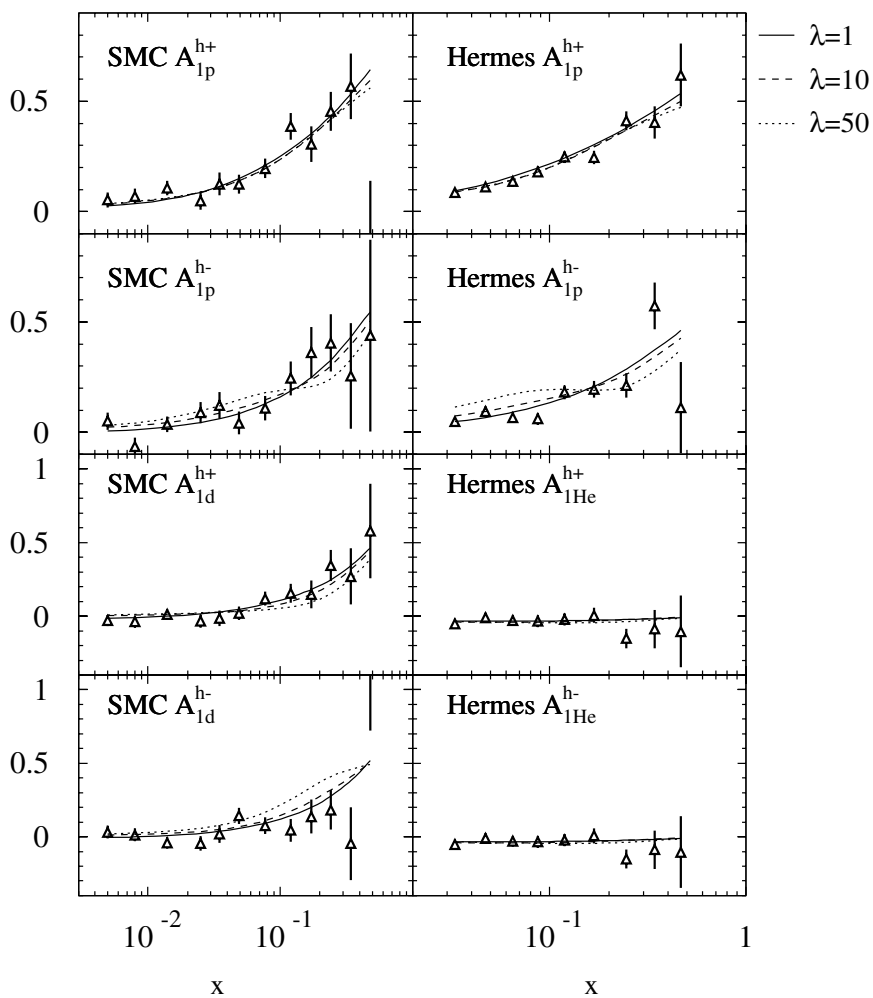

Fig. 4. Fit to SIDIS data $\left(\lambda_{i}=1\right)$ together with the $\sigma_{p}^{h+}$ driven fits 


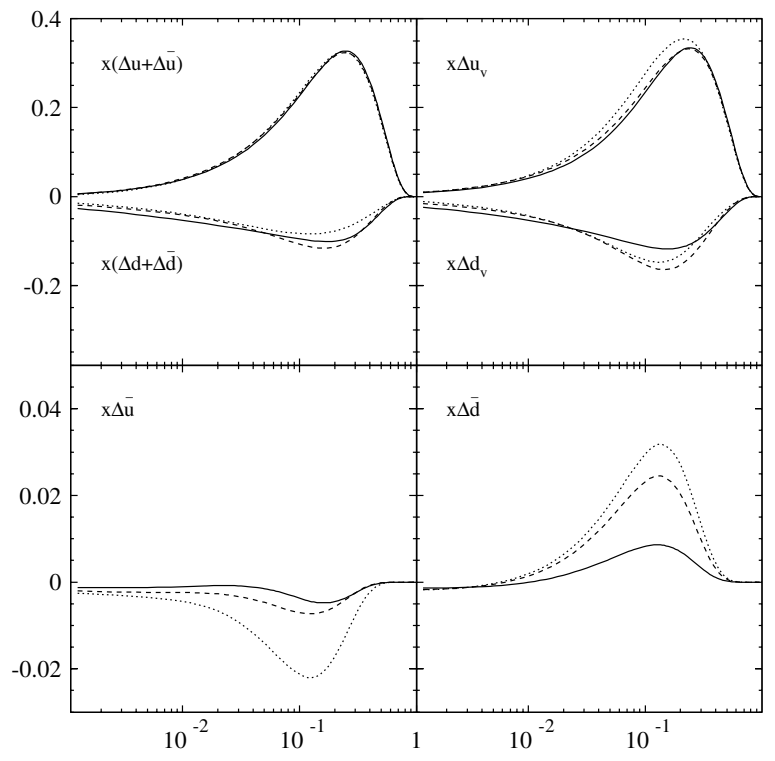

(a)

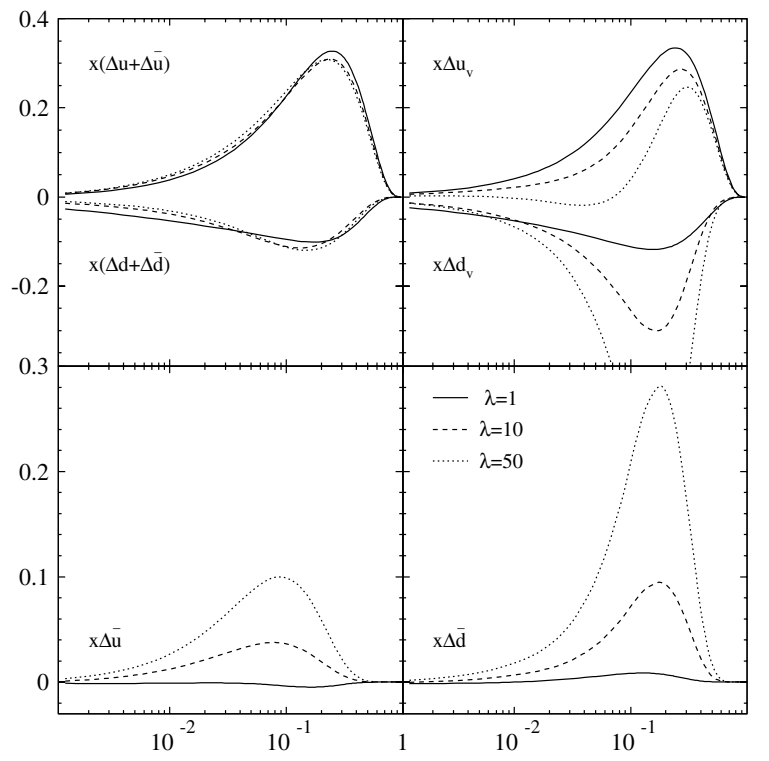

(b)

Fig. 5a,b. Parton densities from $\mathbf{a} \sigma_{p}^{h-}$ and $\mathbf{b} \sigma_{p}^{h+}$ driven fits at $Q^{2}=5.0 \mathrm{GeV}^{2}$

Table 3. $\chi_{i}^{2}(\lambda)$ and $\chi_{\text {tot }}^{2}(\lambda)$ for different SIDIS data sets

\begin{tabular}{|c|c|c|c|c|c|c|}
\hline & \multicolumn{2}{|c|}{$\lambda=1$} & \multicolumn{2}{|c|}{$\lambda=10$} & \multicolumn{2}{|c|}{$\lambda=20$} \\
\hline & $\chi_{i}^{2}$ & $\chi_{\text {tot }}^{2}$ & $\chi_{i}^{2}$ & $\chi_{\text {tot }}^{2}$ & $\chi_{i}^{2}$ & $\chi_{\text {tot }}^{2}$ \\
\hline$\sigma_{p}^{h+}$ & 25.61 & & 21.30 & 360.96 & 20.94 & 450.20 \\
\hline$\sigma_{p}^{h-}$ & 26.93 & & 25.57 & 332.89 & 25.53 & 340.10 \\
\hline$\sigma_{d}^{h+}$ & 5.18 & 319.69 & 3.99 & 385.29 & 3.72 & 504.24 \\
\hline$\sigma_{d}^{h-}$ & 14.71 & & 12.80 & 328.27 & 12.73 & 373.58 \\
\hline$\sigma_{h e}^{h+}$ & 5.39 & & 5.36 & 319.79 & 5.25 & 329.17 \\
\hline$\sigma_{h e}^{h-}$ & 6.69 & & 6.46 & 338.18 & 5.71 & 409.15 \\
\hline
\end{tabular}

and a saturation value outside the $\sqrt{2 N}$ range. SIDIS data are mainly dominated by proton target data, but while $\sigma_{p}^{h-}$ driven fits lead to asymmetries in agreement with other data sets, those driven by $\sigma_{p}^{h+}$ show an increasing disagreement with them, as can be seen in Fig. 4.

The inconsistency between these two data sets can also be seen in the parton distributions they produce, as shown in Fig. 5a,b, for $\sigma_{p}^{h-}$ and $\sigma_{p}^{h+}$ driven fits at $Q^{2}=5.0 \mathrm{GeV}^{2}$, respectively. Notice that the $(\Delta u+\Delta \bar{u})$ and $(\Delta d+\Delta \bar{d})$ distributions, that should be fixed mainly by inclusive data, have minor, although not negligible, changes in the fits driven by either data sets, showing the degree of consistency between inclusive and semi-inclusive data. However, the sea quark distributions depend strongly on which data set has received additional weight. The discrepancy is particularly strong for $\Delta \bar{u}$, which even changes sign.

An interesting feature of SIDIS data is that for all subsets the SIDIS driven fits exceed the $\sqrt{2 N}$ range for $\chi_{\text {tot }}^{2}$ with values of $\lambda$ considerably smaller than the ones typical of inclusive data, as Fig. 3 shows. While inclusive data sets allow $\lambda_{i}$ values of 100 or more with a few units change in $\chi_{\text {tot }}^{2}$, the SIDIS data exceed the allowed range for $\lambda>20$ or even less in the case of $\sigma^{h+}$. In other words, at variance with what happens to DIS data, fits forced to reproduce the SIDIS data lead to considerably poor global fits. This can be interpreted as a weaker level of consistency in the analysis of SIDIS data than in the inclusive case. In Table 3 we present $\chi_{i}^{2}$ values obtained for each data set in the standard fit $\left(\lambda_{i}=1\right)$ and increasing the weights.

In addition to the issue of the accuracy of the data, the analysis of SIDIS experiments relies also on our knowledge of the unpolarized fragmentation functions. Although most of the uncertainties coming from these functions cancel when computing asymmetries, the weaker degree of consistency, and the odd behavior of $\sigma^{h+}$ regardless of whether one uses proton or deuterium targets, and in different experiments, may hint at a failure in the extraction of fragmentation functions, particularly in the more troublesome discrimination between those for positive and negative final state hadrons.

\section{Conclusions}

We have assessed the internal consistency in a NLO analysis of different sets of polarized DIS and SIDIS data. For the inclusive data, the agreement shown between theory and data from the standpoint of the hypothesis testing criterion is confirmed when a detailed analysis using a variant of the parameter fitting criterion is performed. For SIDIS data the level of consistency is considerably weaker, particularly in the case of positively charged final state hadron data. Even though this kind of analysis cannot establish whether either data or some particular ingredients in the theoretical approach are responsible for the discrepancy, the unexpected features found in the analysis of the $\sigma^{h+}$ data, for different targets and coming from different experiments, hint at an inaccuracy in the separation between 
Table A1. Parameters for the $\lambda=1$ fit

\begin{tabular}{ccc}
\hline Parameter & DIS fit & SIDIS fit \\
\hline$\epsilon_{\mathrm{Bj}}$ & -0.002 & -0.004 \\
$\epsilon_{S U(3)}$ & 0.085 & 0.088 \\
$\alpha_{u}$ & 0.858 & 0.858 \\
$\beta_{u}$ & 3.200 & 3.200 \\
$\gamma_{u}$ & 14.929 & 14.969 \\
$\delta_{u}$ & 1.004 & 1.005 \\
$\alpha_{d}$ & 0.434 & 0.433 \\
$\beta_{d}$ & 4.050 & 4.050 \\
$\gamma_{d}$ & 13.888 & 13.939 \\
$\delta_{d}$ & 1.651 & 1.651 \\
$N_{s}$ & -0.074 & -0.075 \\
$\alpha_{\mathrm{s}}$ & 2.500 & 2.491 \\
$\beta_{s}$ & 10.000 & 10.000 \\
$N_{g}$ & 0.239 & 0.238 \\
$\alpha_{g}$ & 1.499 & 1.499 \\
$\beta_{g}$ & 6.000 & 6.000 \\
$N_{\bar{u}}$ & - & -0.014 \\
$\alpha_{\bar{u}}$ & - & 2.311 \\
$\beta_{\bar{u}}$ & - & 7.646 \\
$N_{\bar{d}}$ & - & 0.014 \\
$\alpha_{\bar{d}}$ & - & 2.315 \\
$\beta_{\bar{d}}$ & - & 7.646 \\
\hline
\end{tabular}

positively and negatively charged hadron fragmentation functions.

Acknowledgements. We warmly acknowledge D. de Florian, A. Daleo and C. A. García Canal for helpful comments and discussions.

\section{Appendix: Parameters of the fit}

We present here the parameters of the fit with $\lambda=1$, for the first stage, where we only deal with the inclusive data (DIS fit), and the second stage, where the semi-inclusive data were included (SIDIS fit).

\section{References}

1. J. Ashman et al., EMC Collab., Nucl. Phys. B 328, 1 (1989)

2. B. Lampe, E. Reya, Phys. Rep. 332, 1 (2000); E. Leader et al., Phys, Rep. 261, 1 (1995)

3. T. Gehrmann, W.J. Stirling, Phys. Rev. D 53, 6100 (1996); R.D. Ball, S. Forte, G. Ridolfi, Nucl. Phys. B 496, 337 (1997); C. Bourrely, F. Buccella, O. Pisanti, P. Santorelli, J. Soffer, Prog. Theor. Phys. 99, 1017 (1998); E. Leader, A.V. Sidorov, D.B. Stamenov, Int. J. Mod. Phys. A 13, 5573 (1998); Phys. Rev. D 58, 114028 (1998); Y. Goto et al., Phys. Rev. D 62, 034017 (2000); M. Glück, E. Reya, M. Stratmann, W. Vogelsang, Phys. Rev. D 63, 094005 (2001); J. Blumlein, H. Bottcher, Nucl. Phys. B 636, 225 (2002); J. Bartelski, S. Tatur, hep-ph/0205089
4. D. de Florian, O.A. Sampayo, R. Sassot, Phys. Rev. D 57, 5803 (1998)

5. D. de Florian, R. Sassot, Phys. Rev. D 62, 094025 (2000)

6. M. Stratmann, W. Vogelsang, Phys. Rev. D 64, 114007 (2001)

7. E. Christova, E. Leader, Nucl. Phys. 607, 369 (2001)

8. M. Glück, E. Reya, hep-ph/0203063

9. A. Kotzinian, hep-ph/0211162 v2

10. M. Wakamatsu, T. Watabe, Phys. Rev. D 62, 017506 (2000); B. Dressler et al., Eur. Phys. J. C 14, 147 (2000); M. Glück, E. Reya, Mod. Phys. Lett. A 15, 883 (2000); R.S. Bhalerao, Phys. Rev. C 63, 025208 (2001); T. Morii, T. Yamanishi, Riken Rev. 28, 118 (2000); D.K. Ghosh et al., Phys. Rev. D 62, 094012 (2000); M. Glück, A. Hartle, E. Reya, Eur. Phys. J. C 19, 77 (2001); C. Bourrely, J. Soffer, F. Buccella, Eur. Phys. J. C 23, 487 (2002)

11. E.A. Hawker et al., Phys. Rev. Lett. 80, 3715 (1998); R.S. Towell et al., Phys. Rev. D 64, 052002 (2001)

12. N. Bianchi, R. Jakob, hep-ph/0108078

13. B. Adeva et al., SMC Collab., Phys. Lett. B 369, 93 (1996); 420, 180 (1998)

14. K. Ackerstaff et al., HERMES Collab., Phys. Lett. B 464, 123 (1999)

15. J.C. Collins, J. Pumplin, hep-ph/0105207

16. A. Daleo, C.A. Garcia Canal, G.A. Navarro, R. Sassot, Int. J. Mod. Phys. A 17, 269 (2002)

17. R. Mertig, W.L. van Neerven, Z. Phys. C 70, 637 (1996); W. Vogelsang, Phys. Rev. D 54, 20023 (1996)

18. M. Gück, E. Reya, W. Vogelsang, Phys. Rev. D 53, 6100 (1996)

19. D. Stump et al., hep-ph/0101051

20. D. de Florian, C.A. Garcia Canal, R. Sassot, Nucl. Phys. B 470, 195 (1996)

21. D. de Florian, M. Stratmann, W. Vogelsang, Phys. Rev. D 57, 5811 (1998)

22. J.D. Bjorken, Phys. Rev. 148, 1467 (1966)

23. M. Glück, E. Reya, A. Vogt, Eur. Phys. J. C 5, 461 (1998)

24. S. Kretzer, Phys. Rev. D 62, 054001 (2000)

25. S. Kretzer, E. Leader, E. Christova, Eur. Phys. J. C 22, 269 (2001)

26. J. Ellis, R.L. Jaffe, Phys. Rev. D 9, 1444 (1974)

27. B. Adeva et al., SMC Collab., Phys. Rev. D 58, 112001 (1998)

28. K. Abe et al., E143 Collab., Phys. Rev. D 58, 112003 (1998)

29. P.L. Anthony et al., E155 Collab., Phys. Lett. B 463, 339 (1999); G.S. Mitchell, Ph.D. Thesis University of Wisconsin-Madison, SLAC-Report-540 (1999)

30. P.L. Anthony et al., E142 Collab., Phys. Rev. D 54, 6620 (1996)

31. K. Abe et al., E154 Collab., Phys. Rev. Lett. 79, 26 (1997); Phys. Lett. B 405, 180 (1997)

32. R. Thorne, hep-ph/0211113 